\title{
FUNCTORIAL PROLONGATIONS OF LIE GROUPOIDS
}

\author{
IVAN KOLÁR̆ \\ Department of Algebra and Geometry \\ Faculty of Science, Masaryk University \\ Janáčkovo nám. 2a, 602 00 Brno, Czech Republic \\ E-mail: kolar@math.muni.cz
}

\begin{abstract}
For every Lie groupoid $\Phi$ with $m$-dimensional base $M$ and every fiber product preserving bundle functor $F$ on the category of fibered manifolds with $m$-dimensional bases and fiber preserving maps with local diffeomorphisms as base maps, we construct a Lie groupoid $\mathcal{F} \Phi$ over $M$. Every action of $\Phi$ on a fibered manifold $Y \rightarrow M$ is extended to an action of $\mathcal{F} \Phi$ on $F Y \rightarrow M$.
\end{abstract}

Charles Ehresmann introduced the $r$-th prolongation $\Phi^{r}$ of an arbitrary Lie groupoid $\Phi$ and clarified that if $\Phi$ acts on a fibered manifold $Y$, then $\Phi^{r}$ acts canonically on the $r$-th jet prolongation $J^{r} Y$ of $Y$, [2]. These ideas are of fundamental importance for the general theory of geometric object fields and for the theory of natural bundles and natural operators, [9]. The principal bundle form of the Ehresmann's construction was developed by P. Libermann, [10], and the author, [9]. The theory of principal prolongations of principal bundles has found important applications even in the gauge theories of mathematical physics, see e.g. [4].

The main purpose of the present paper is to generalize the Ehresmann's construction to an arbitrary fiber product preserving bundle functor $F$ on the category $\mathcal{F} \mathcal{M}_{m}$ of fibered manifolds with $m$-dimensional bases and fiber preserving maps with local diffeomorphisms as base maps. The functor $J^{r}$ of $r$-th jet prolongation is the best known special case. Our approach is essentially based on a complete characterization of $F$ in terms of Weil algebras, [8].

2000 Mathematics Subject Classification: 58A20, 58A32.

Key words and phrases: Lie groupoid, fiber product preserving bundle functor, functorial prolongation, Weil algebra.

The author was supported by the Ministery of Education of the Czech Republic under the project MSM 0021622409 and the grant of GAČR No. 201/05/0523.

The paper is in final form and no version of it will be published elsewhere. 
In Section 1 we recall the basic concepts and the original construction by Ehresmann. Section 2 is devoted to the classical interpretation of the product preserving bundle functors on the category $\mathcal{M f}$ of all manifolds and all smooth maps as the $A$-velocities functors $T^{A}$, where $A$ is an arbitrary Weil algebra, [9]. One sees immediately, that the $T^{A}$-prolongation of Lie groupoid $\Phi$ over $M$ is a Lie groupoid $T^{A} \Phi$ over $T^{A} M$ and every action of $\Phi$ on a fibered manifold $p: Y \rightarrow M$ is canonically extended into an action of $T^{A} \Phi$ on the fibered manifold $T^{A} p: T^{A} Y \rightarrow T^{A} M$. In Section 3 we recall the description of a fiber product preserving bundle functor $F$ on $\mathcal{F M}_{m}$ in terms of Weil algebras, [8]. This enables us to introduce, in Section 4, the $F$-prolongation $\mathcal{F} \Phi$ of every Lie groupoid $\Phi$. This is also a Lie groupoid over the same base. Then we compare this construction with the principal $F$-prolongation of a principal bundle defined in [1]. In Section 5 we discuss in detail the basic examples, namely the $r$-th jet prolongation $J^{r}$, the vertical $A$-functor $V^{A}$ and the $r$-th vertical jet prolongation $J^{r, v}$. Section 6 is devoted to $F$-prolongations of actions. Finally we remark that our ideas can be applied to smooth categories as well.

All manifolds and maps are assumed to be infinitely differentiable. Unless otherwise specified, we use the terminology and notations from [9].

1. In the algebraic sense, a groupoid is a category in which all elements are invertible. We write $a$ or $b$ for the right or left unit map (also called source or target), respectively. A smooth groupoid $\Phi \underset{b}{\stackrel{a}{\longrightarrow}} M$ is a groupoid such that $\Phi$ and $M$ are manifolds, both $a$, $b: \Phi \rightarrow M$ are surjective submersions and the partial composition law

$$
\varkappa: \Phi^{a} \times_{M} \Phi^{b} \rightarrow \Phi, \quad \Phi^{a}:=(\Phi \stackrel{a}{\rightarrow} M), \quad \Phi^{b}:=(\Phi \stackrel{b}{\rightarrow} M)
$$

as well as the unit injection $e: M \rightarrow \Phi$ are smooth maps. The product groupoid is of the form

$$
M \times G \times M,
$$

where $M$ is a manifold, $G$ is a Lie group, $a=p r_{3}, b=p r_{1}, e(x)=\left(x, e_{G}, x\right)$, where $e_{G}$ is the unit of $G$, and

$$
\varkappa\left(\left(x_{3}, g_{2}, x_{2}\right)\left(x_{2}, g_{1}, x_{1}\right)\right)=\left(x_{3}, g_{2} g_{1}, x_{1}\right),
$$

the product $g_{2} g_{1}$ being in $G$. A smooth groupoid is called a Lie groupoid, if it is locally isomorphic to the product one, [11].

For every Lie groupoid $\Phi$ and every $x \in M$,

$$
\Phi_{x}:=\{\theta \in \Phi, a \theta=x\}
$$

is a principal bundle, whose structure group $G_{x}$ is the isotropy group of $\Phi$ over $x$. Conversely, if $P(M, G)$ is a principal bundle, then the space of all equivalence classes

$$
P P^{-1}=P \times P / \sim, \quad(v, u) \sim(v g, u g), \quad u, v \in P, g \in G,
$$

is a Lie groupoid over $M$ with respect to the composition $\{w, v\}\{v, u\}=\{w, u\}$. We write $v u^{-1}$ for the equivalence class determined by $(v, u) \in P \times P$. In the product case, $(M \times G \times M)_{x}$ is the product principal bundle $M \times G$. Conversely, $(M \times G)(M \times G)^{-1}=$ $M \times G \times M$. 
An action of a Lie groupoid $\Phi$ on a fibered manifold $p: Y \rightarrow M$ is a map $\lambda$ : $\Phi^{a} \times_{M} Y \rightarrow Y$ such that

$$
p(\lambda(\theta, y))=b(\theta), \lambda\left(\varkappa\left(\theta_{2}, \theta_{1}\right), y\right)=\lambda\left(\theta_{2}, \lambda\left(\theta_{1}, y\right)\right), \lambda(e(x), y)=y .
$$

Every left group action $l: G \times S \rightarrow S$ induces an action of $M \times G \times M$ on the product fibered manifold $M \times S \rightarrow M$ by

$$
\lambda\left(\left(x_{2}, g, x_{1}\right),\left(x_{1}, s\right)\right)=\left(x_{2}, l(g, s)\right) .
$$

In the principal bundle form, $M \times S$ is a fiber bundle associated to $M \times G$.

Ehresmann introduced the $r$-th prolongation $\Phi^{r}$ of a Lie groupoid $\Phi$ as follows, [2]. The elements of $\Phi^{r}$ are $r$-jets $j_{x}^{r} \sigma$, where $\sigma$ is a local section $U \rightarrow \Phi^{a}$ such that $b \circ \sigma: U \rightarrow M$ is a local diffeomorphism. The composition $\varkappa^{r}$ is defined by

$$
\varkappa^{r}\left(j_{\bar{x}}^{r} \bar{\sigma}, j_{x}^{r} \sigma\right)=j_{x}^{r} \varkappa(\bar{\sigma} \circ b \sigma, \sigma), \quad \bar{x}=b(\sigma(x)) .
$$

We write $a_{r}, b_{r}:=\Phi^{r} \rightarrow M$ and $e_{r}: M \rightarrow \Phi^{r}$ for the source and target projections and the unit injection of $\Phi^{r}$. For every action $\lambda: \Phi^{a} \times_{M} Y \rightarrow Y$, the induced action

$$
\lambda^{r}:\left(\Phi^{r}\right)^{a_{r}} \times_{M} J^{r} Y \rightarrow J^{r} Y
$$

is defined by

$$
\lambda^{r}\left(j_{x}^{r} \sigma, j_{x}^{r} s\right)=j_{\bar{x}}^{r}\left(\lambda(\sigma, s) \circ(b \sigma)^{-1}\right), \quad \bar{x}=b(\sigma(x)),
$$

where $\sigma$ or $s$ is a local section of $\Phi^{a}$ or $Y$, respectively, [2].

The main problem of the present paper is to extend the Ehresmann's construction to an arbitrary fiber product preserving bundle functor $F$ on $\mathcal{F} \mathcal{M}_{m}$. We underline that we cannot apply $F$ to $\varkappa$, for $\varkappa$ is not an $\mathcal{F} \mathcal{M}_{m}$-morphism.

2. But we can use the description of $F$ in terms of Weil algebras deduced in [8].

The space $\mathbb{D}_{k}^{r}=J_{0}^{r}\left(\mathbb{R}^{k}, \mathbb{R}\right)$ is an algebra with respect to the addition and multiplication induced by the addition and multiplication of reals. A Weil algebra $A$ can be defined as a factor algebra of $\mathbb{D}_{k}^{r}$. Algebra $A$ defines a bundle functor $T^{A}$ on $\mathcal{M} f$ in such a way that $T^{\mathbb{D}_{k}^{r}}=T_{k}^{r}$ is the classical functor of $(k, r)$-velocities, [9], [7]. The elements of $T^{A} M$ are called $A$-velocities on $M$ and are written as

$$
j^{A} \gamma, \quad \gamma: \mathbb{R}^{k} \rightarrow M
$$

For every smooth map $f: M \rightarrow N, T^{A} f: T^{A} M \rightarrow T^{A} N$ is defined by $T^{A} f\left(j^{A} \gamma\right)=$ $j^{A}(f \circ \gamma)$. A fundamental result reads that all product preserving bundle functors on $\mathcal{M} f$ are the Weil functors. Moreover, if $A$ and $B$ are two Weil algebras, then the natural transformations $T^{A} \rightarrow T^{B}$ are in bijection with the algebra homomorphisms $\mu: A \rightarrow B$, [9]. We write $\mu_{M}: T^{A} M \rightarrow T^{B} M$ for the value of the natural transformation on $M$.

One verifies easily that for every Lie groupoid $\Phi \underset{b}{\stackrel{a}{\longrightarrow}} M, T^{A} \Phi \underset{T^{A} b}{\stackrel{T^{A} a}{\longrightarrow}} T^{A} M$ is also a Lie groupoid with partial composition $T^{A} \varkappa$ and unit injection $T^{A} e: T^{A} M \rightarrow T^{A} \Phi$. If $\lambda: \Phi^{a} \times_{M} Y \rightarrow Y$ is an action of $\Phi$ on $Y$, then $T^{A} \lambda:\left(T^{A} \Phi\right)^{T^{A} a} \times_{T^{A} M} T^{A} Y \rightarrow T^{A} Y$ is an action of $T^{A} \Phi$ on $T^{A} Y \rightarrow T^{A} M$. 
3. We recall that two $\mathcal{F} \mathcal{M}$-morphisms $f, g: Y \rightarrow \bar{Y}$ with base maps $\underline{f}, \underline{g}: M \rightarrow \bar{M}$ determine the same $(q, s, r)$-jet at $y \in Y$ (denoted by $\left.j_{y}^{q, s, r} f\right), s \geq q \leq r, x=p(y)$, if

$$
j_{y}^{q} f=j_{y}^{q} g, \quad j_{y}^{s}\left(f \mid Y_{x}\right)=j_{y}^{s}\left(g \mid Y_{x}\right), \quad j_{x}^{r} \underline{f}=j_{x}^{r} \underline{g} .
$$

A bundle functor $F$ on $\mathcal{F M}$ is said to be of the order $(q, s, r)$, if

$$
j_{y}^{q, s, r} f=j_{y}^{q, s, r} g \quad \text { implies } \quad F f\left|F_{y} Y=F g\right| F_{y} Y .
$$

We say that $r$ is the base order of $F$.

Even the fiber product preserving bundle functors on $\mathcal{F} \mathcal{M}_{m}$ can be characterized by means of Weil algebras. The basic examples of such functors are the $r$-th jet prolongation $J^{r}$, the vertical Weil functor

$$
V^{A} Y=\bigcup_{x \in M} T^{A}\left(Y_{x}\right) \quad \text { with } T^{A} f \text { defined fiberwise, }
$$

the $r$-th vertical jet prolongation

$$
J^{r, v} Y=\bigcup_{x \in M} J_{x}^{r}\left(M, Y_{x}\right) \quad \text { with } J^{r, v} f \text { defined fiberwise, }
$$

their iterations (e.g. the $r$-th nonholonomic jet prolongation $\widetilde{J}^{r}$ ) and subfunctors (e.g. the $r$-th semiholonomic jet prolongation $\bar{J}^{r}$ ).

According to [8], every such functor $F$ of the base order $r$ is identified with a triple $(A, H, t)$, where $A$ is a Weil algebra, $H: G_{m}^{r} \rightarrow$ Aut $A$ is a group homomorphism and $t: \mathbb{D}_{m}^{r} \rightarrow A$ is an equivariant algebra homomorphism under the identification of the $r$-th jet group $G_{m}^{r}$ in dimension $m$ with Aut $\mathbb{D}_{m}^{r}$ given by $(g, X) \mapsto X \circ g^{-1}, X \in \mathbb{D}_{m}^{r}, g \in G_{m}^{r}$.

Given $A, H$ and $t$, the functor $F=(A, H, t)$ can be reconstructed as follows. In the product case of $M \times N \rightarrow M$,

$$
F(M \times N)=P^{r} M\left[T^{A} N, H_{N}\right]
$$

is the fiber bundle associated to the $r$-th order frame bundle $P^{r} M$ with standard fiber $T^{A} N$ and the action $H_{N}$ of $G_{m}^{r}$ on $T^{A} N$ determined by the natural transformations $H(g)_{N}: T^{A} N \rightarrow T^{A} N$. In the general case of $p: Y \rightarrow M, F Y$ is the subset of $F(M \times Y)=$ $P^{r} M\left[T^{A} Y, H_{Y}\right]$ formed by the equivalence classes $\{u, Z\}$ satisfying

$$
t_{M}(u)=T^{A} p(Z) \in T^{A} M, \quad u \in P^{r} M, Y \in T^{A} Y,
$$

where $t_{M}: T_{m}^{r} M \rightarrow T^{A} M$ and $P^{r} M \subset T_{m}^{r} M$. For an $\mathcal{F} \mathcal{M}_{m}$-morphism $f: Y \rightarrow \bar{Y}$ over $\underline{f}: M \rightarrow \bar{M}$, we have

$$
F f=\left\{P^{r} \underline{f}, T^{A} f\right\},
$$

where $P^{r} \underline{f}: P^{r} M \rightarrow P^{r} \bar{M}$ and $T^{A} f: T^{A} Y \rightarrow T^{A} \bar{Y}$ form a morphism of associated bundles.

4. Consider the $F$-prolongation of $\Phi \stackrel{a}{\rightarrow} M$ and write $\pi: F\left(\Phi^{a}\right) \rightarrow \Phi^{a}$ for the bundle projection and $\bar{a}=a \circ \pi, \bar{b}=b \circ \pi: F \Phi^{a} \rightarrow M$. Let $\Pi^{r} M \underset{\beta}{\stackrel{\alpha}{\longrightarrow}} M$ be the groupoid of all invertible $r$-jets on $M$. Hence $(\bar{a}, \bar{b}): F \Phi^{a} \rightarrow M \times M$ and $(\alpha, \beta): \Pi^{r} M \rightarrow M \times M$, so that we can construct $\Pi^{r} M \times_{M \times M} F \Phi^{a}$. 
Definition 1. The $F$-prolongation of a Lie groupoid $\Phi$ is the subset

$$
\mathcal{F} \Phi \subset \Pi^{r} M \times_{M \times M} F \Phi^{a}
$$

of all pairs $\left(v \circ u^{-1},\{u, Z\}\right)$ satisfying $t_{M} v=T^{A} b(Z)$.

Hence the elements of $\mathcal{F} \Phi$ are the equivalence classes, with respect to the action of $G_{m}^{r}$

$$
\{v, Z, u\} \quad \text { satisfying } \quad T^{A} a(Z)=t_{M} u, T^{A} b(Z)=t_{M} v,
$$

$u, v \in P^{r} M, Z \in T^{A} \Phi$. Given another $\{w, \bar{Z}, v\} \in \mathcal{F} \Phi$, we define the composition by

$$
\{w, \bar{Z}, v\} *\{v, Z, u\}=\left\{w, T^{A} \varkappa(\bar{Z}, Z), u\right\} .
$$

Write $\widetilde{a}, \widetilde{b}: \mathcal{F} \Phi \rightarrow M$ for the projections determined by (10). Define $\widetilde{e}: M \rightarrow \mathcal{F} \Phi$ by $\widetilde{e}(x)=\left\{u, j^{A} \widehat{e(x)}, u\right\}$, where $\widehat{ }$ denotes the constant map of $\mathbb{R}^{k}$ into $e(x)$. Then one verifies directly

Proposition 1. $\mathcal{F} \Phi \underset{\widetilde{b}}{\stackrel{\widetilde{a}}{\longrightarrow}} M$, with the partial composition law (12) and the unit injection $\widetilde{e}$ is a Lie groupoid over $M$.

We remark that the Lie algebroid of $\mathcal{F} \Phi$ was constructed in the principal bundle form in $[6]$.

We recall $\Pi^{r} \mathbb{R}^{m}=\mathbb{R}^{m} \times G_{m}^{r} \times \mathbb{R}^{m}$. In the product case of $\Phi=\mathbb{R}^{m} \times G \times \mathbb{R}^{m}$, we have

$$
\mathcal{F} \Phi=\mathbb{R}^{m} \times G_{m}^{r} \times T^{A} G \times \mathbb{R}^{m} .
$$

In [1], the principal $F$-prolongation $W^{F} P$ of a principal bundle $P(M, G)$ is defined by

$$
W^{F} P=P^{r} M \times_{M} F P .
$$

This is a principal bundle over $M$, whose structure group is the semidirect product $W_{H}^{A} G=G_{m}^{r} \rtimes T^{A} G$ with the multiplication

$$
\left(g_{1}, X_{1}\right)\left(g_{2}, X_{2}\right)=\left(g_{1} \circ g_{2}, H_{G}\left(g_{2}^{-1}\right)\left(X_{1}\right) \cdot X_{2}\right),
$$

the product $\cdot$ being in $T^{A} G$. Using (13), one verifies easily that

$$
\mathcal{F}\left(P P^{-1}\right)=\left(W^{F} P\right)\left(W^{F} P\right)^{-1} .
$$

5. We are going to discuss the basic examples from Section 3.

ExAmPLE 1 . For $F=J^{r}$, we have $A=\mathbb{D}_{m}^{r}, H=\mathrm{id}_{G_{m}^{r}}, t=\mathrm{id}_{\mathbb{D}_{m}^{r}}$, so that $t_{M}=\mathrm{id}_{T_{m}^{r} M}$. Hence the elements of $J^{r} Y$ can be interpreted as the equivalence classes

$$
\{u, Z\}, \quad u \in P^{r} M, \quad Z \in T_{m}^{r} Y \quad \text { satisfying } T_{m}^{r} p(Z)=u .
$$

In this situation, $Z \circ u^{-1}=j_{x}^{r} s$, where $s$ is a local section of $Y$. The elements of $\mathcal{J}^{r} \Phi$ are the equivalence classes

$$
\{v, Z, u\}, \quad Z \in T_{m}^{r} \Phi, \quad u=T_{m}^{r} a(Z), \quad v=T_{m}^{r} b(Z) .
$$


We have $Z \circ u^{-1}=j_{x}^{r} \sigma$, where $\sigma$ is a local section of $\Phi^{a}$. Then $T_{m}^{r} b(Z)=v$ implies $v \circ u^{-1}=j_{x}^{r}(b \circ \sigma)$. So our set $\mathcal{J}^{r} \Phi$ and the set $\Phi^{r}$ by Ehresmann coincide. Consider another $\{w, \bar{Z}, v\} \in \mathcal{J}^{r} \Phi$. Then (12) yields

$$
\{w, \bar{Z}, v\} *\{v, Z, u\}=\left\{w, T_{m}^{r} \varkappa(\bar{Z}, Z), u\right\} .
$$

Writing $\bar{Z} \circ v^{-1}=j_{\bar{x}}^{r} \bar{\sigma}$, we find $T_{m}^{r} \varkappa(\bar{Z}, Z) \circ u^{-1}=T_{m}^{r} \varkappa\left(\bar{Z} \circ v^{-1} \circ v \circ u^{-1}, Z \circ u^{-1}\right)=$ $j_{x}^{r} \varkappa(\bar{\sigma} \circ b \sigma, \sigma)$. So even the composition in $\mathcal{J}^{r} \Phi$ coincides with that one introduced by Ehresmann.

ExAmple 2. Consider the vertical Weil functor $F=V^{A}$. The base order of $V^{A}$ is 0 , $P^{0} M=M$ and $G_{m}^{0}$ is the one-element group. Further, $\mathbb{D}_{m}^{0}=\mathbb{R}$ and $t$ is the zero homomorphism. Hence

$$
\mathcal{V}^{A} \Phi=\left\{Z \in T^{A} \Phi, T^{A} a(Z)=j^{A} \widehat{x}_{1}, T^{A} b(Z)=j^{A} \widehat{x}_{2}\right\},
$$

where $x_{1}=\widetilde{a}(Z), x_{2}=\widetilde{b}(Z)$. In other words, the elements of $\mathcal{V}^{A} \Phi$ are of the form

$$
j^{A} \varphi(\tau), \quad a \varphi(\tau)=x_{1}, \quad b \varphi(\tau)=x_{2} \quad \text { for all } \quad \tau \in \mathbb{R}^{k} .
$$

For another $\bar{Z} \in \mathcal{V}^{A} \Phi, \bar{Z}=j^{A} \psi(\tau), a \psi(\tau)=x_{2}, b \psi(\tau)=x_{3}$, the composition $\varkappa(\psi(\tau), \varphi(\tau))$ is defined for all $\tau \in \mathbb{R}^{k}$ and we have

$$
\bar{Z} * Z=j^{A} \varkappa(\psi(\tau), \varphi(\tau)) .
$$

EXAmple 3 . In the case $F=J^{r, v}$, we have $A=\mathbb{D}_{m}^{r}, H=\mathrm{id}_{G_{m}^{r}}$, and $t: \mathbb{D}_{m}^{r} \rightarrow \mathbb{D}_{m}^{r}$ is the zero homomorphism. Hence for every $u \in P_{x}^{r} M$ we have $t_{M} u=j_{0}^{r} \widehat{x}$. So the elements of $J^{r, v} Y$ can be interpreted as the equivalence classes

$$
\{u, Z\} \text { satisfying } T_{m}^{r} p(Z)=j_{0}^{r} \widehat{x},
$$

$u \in P_{x}^{r} M, Z \in T_{m}^{r} Y$. In this situation, $Z \circ u^{-1}=j_{x}^{r} s$, where $s$ is a local map of $M$ into $Y_{x}$. Write

$$
\Phi_{x_{2}, x_{1}}=\left\{\theta \in \Phi, a \theta=x_{1}, b \theta=x_{2}\right\} .
$$

For every manifold $N, \varkappa$ induces a map

$$
\begin{aligned}
& \tilde{\varkappa}_{N}: J^{r}\left(N, \Phi_{x_{2}, x_{3}}\right) \times_{N} J^{r}\left(N, \Phi_{x_{1}, x_{2}}\right) \rightarrow J^{r}\left(N, \Phi_{x_{1}, x_{3}}\right), \\
& \tilde{\varkappa}_{N}\left(j_{z}^{r} \psi(\tau), j_{z}^{r} \varphi(\tau)\right)=j_{z}^{r} \varkappa(\psi(\tau), \varphi(\tau)), \quad z \in N .
\end{aligned}
$$

Having $\{v, Z, u\} \in \mathcal{J}^{r, v} \Phi$, the conditions $T_{m}^{r} a(Z)=j_{0}^{r} \widehat{x}_{1}, T_{m}^{r} b(Z)=j_{0}^{r} \widehat{x}_{2}$ are equivalent to $Z \in T_{m}^{r} \Phi_{x_{1}, x_{2}}$. Hence the elements of $\mathcal{J}^{r, v} \Phi$ are the pairs

$$
(X, S), \quad X \in \Pi^{r} M, S \in J_{x_{1}}^{r}\left(M, \Phi_{x_{1}, x_{2}}\right), \quad x_{1}=\alpha X, x_{2}=\beta X .
$$

Consider another $(\bar{X}, \bar{S}) \in \mathcal{J}^{r, v} \Phi$ of the form $\{w, \bar{Z}, v\}$. Formally, the product is defined by the same formula (16). But now we have

$$
T_{m}^{r} \varkappa(\bar{Z}, Z) \circ u^{-1}=\tilde{\varkappa}_{M}\left(\bar{Z} \circ v^{-1} \circ v \circ u^{-1}, Z \circ u^{-1}\right) .
$$

Hence the jet formula for the composition in $\mathcal{J}^{r, v} \Phi$ is

$$
(\bar{X}, \bar{S}) *(X, S)=\left(\bar{X} \circ X, \tilde{\varkappa}_{M}(\bar{S} \circ X, S)\right) .
$$


6. Consider an action $\lambda: \Phi^{a} \times_{M} Y \rightarrow Y$.

Definition 2. We define $\mathcal{F} \lambda:(F \Phi)^{\widetilde{a}} \times_{M} F Y \rightarrow F Y$ by

$$
\mathcal{F} \lambda(\{v, Z, u\},\{u, Q\})=\left\{v, T^{A} \lambda(Z, Q)\right\},
$$

$u, v \in P^{r} M, Z \in T^{A} \Phi, Q \in T^{A} Y$.

Analogously to Section 4, one verifies directly

Proposition 2. $\mathcal{F} \lambda$ is an action of $\mathcal{F} \Phi$ on $F Y \rightarrow M$.

In Example 1, if $\{v, Z, u\} \in \mathcal{J}^{r} \Phi$ and $\{u, Q\} \in J^{r} Y$ so that $Z \circ u^{-1}=j_{x}^{r} \sigma, v \circ u^{-1}=$ $j_{x}^{r}(b \circ \sigma)$ and $Q \circ u^{-1}=j_{x}^{r} s \in J^{r} Y$, then $T_{m}^{r} \lambda(Z, Q) \circ v^{-1}=T_{m}^{r} \lambda\left(Z \circ u^{-1}, Q \circ u^{-1}\right) \circ u \circ v^{-1}=$ $j_{\bar{x}}^{r}\left(\lambda(\sigma, s) \circ(b \sigma)^{-1}\right)$. This is the original formula (5) by Ehresmann.

In Example 2, if $S=\left(\bar{x}, j^{A} \varphi(\tau), x\right) \in \mathcal{V}^{A} \Phi$ and $Q=\left(x, j^{A} \psi(\tau)\right) \in V^{A} Y$, then

$$
\mathcal{V}^{A} \lambda(S, Q)=j^{A} \lambda(\varphi(\tau), \psi(\tau))
$$

In Example 3, for every manifold $N$ we have the induced map

$$
\begin{aligned}
& \widetilde{\lambda}_{N}: J^{r}\left(N, \Phi_{x_{1}, x_{2}}\right) \times_{N} J^{r}\left(N, Y_{x_{1}}\right) \rightarrow J^{r}\left(N, Y_{x_{2}}\right), \\
& \tilde{\lambda}_{N}\left(j_{z}^{r}(\sigma, s)\right)=j_{z}^{r} \lambda(\sigma, s), \quad z \in N .
\end{aligned}
$$

Given $\{v, Z, u\} \in \mathcal{J}^{r, v} \Phi$ and $\{u, Q\} \in J^{r, v} Y$, we have

$$
T_{m}^{r} \lambda(Z, Q) \circ v^{-1}=\widetilde{\lambda}_{M}\left(Z \circ u^{-1}, Q \circ u^{-1}\right) \circ\left(u \circ v^{-1}\right) .
$$

Hence the jet formula for $\mathcal{J}^{r, v} \lambda$ is

$$
\mathcal{J}^{r, v} \lambda((X, S), T)=\widetilde{\lambda}_{M}(S, T) \circ X^{-1},
$$

$X \in \Pi^{r} M, S \in J_{x_{1}}^{r}\left(M, \Phi_{x_{1}, x_{2}}\right), T \in J_{x_{1}}^{r}\left(M, Y_{x_{1}}\right)$.

7. We remark that our construction can be applied to every smooth category $C \underset{b}{\stackrel{a}{\longrightarrow}} M$ with the composition $\varkappa: C^{a} \times_{M} C^{b} \rightarrow C,[3],[5]$. (Two simple examples are the category $C E$ of all linear maps between the individual fibers of a vector bundle $E \rightarrow M$ and the category $J^{r}(M, M)$ of all $r$-jets of a manifold $M$ into itself.) One defines $\mathcal{F} C$ as a subset

$$
\mathcal{F} C \subset \Pi^{r} M \times_{M \times M} F C^{a}
$$

analogously to (10). The composition in $\mathcal{F} C$ is introduced by the formula (12).

\section{References}

[1] M. Doupovec and I. Koláŕ, Iteration of fiber product preserving bundle functors, Monatsh. Math. 134 (2001), 39-50.

[2] C. Ehresmann, Les prolongements d'un espace fibré différentiable, CRAS Paris 240 (1955), $1755-1757$.

[3] C. Ehresmann, Prolongements des catégories différentiables, Topologie et Géom. Différentielle 6 (1964), 1-8.

[4] L. Fatibene and M. Francaviglia, Natural and Gauge Natural Formalism for Classical Field Theories, Kluwer, 2003. 
[5] I. Kolár, On the jet prolongations of smooth categories, Bull. de l'Acad. Polonaise des Sciences, Série des sciences math. astr. et phys. 24 (1976), 883-887.

[6] I. Kolář, Functorial prolongations of Lie algebroids, in: Proceedings Conf. Prague 2004, Charles University, Prague, 2005, 301-309.

[7] I. Kolář and A. Cabras, On the functorial prolongations of principal bundles, Comment. Math. Univ. Carolinae 47 (2006), 719-731.

[8] I. Kolář and W. M. Mikulski, On the fiber product preserving bundle functors, Differential Geometry and Its Applications 11 (1999), 105-115.

[9] I. Kolář, P. W. Michor and J. Slovák, Natural Operations in Differential Geometry, Springer-Verlag, 1993.

[10] P. Libermann, Sur les prolongements des fibrés principaux et des groupö̈des différentiables banachiques, Sém. Math. Supérieures 42, Presses Univ. Montreal, 1971, 7-108.

[11] K. Mackenzie, Lie Groupoids and Lie Algebroids in Differential Geometry, Cambridge University Press, 1987. 